\title{
Comet Tago-Sato-Kosaka 1969 IX: Tail Structure 25 December 1969 to 12 January 1970
}

\author{
FREEMAN D. MILLER \\ Department of Astronomy, University of Michigan, Dennison Building, \\ Ann Arbor, Michigan 48109
}

Received October 24, 1977; revised June 5, 1978

\begin{abstract}
On photographs taken between 25 December 1969 and 12 January 1970 with the Michigan Curtis Schmidt on Cerro Tololo and the Hamburg Schmidt at the Boyden Observatory, Comet $1969 \mathrm{IX}$ is seen projected on planes nearly perpendicular to its orbit. During this period the Type I tail was free of major structural disturbances, and the faintness of the Type II tail facilitated study of the Type I tail near the head. Characteristics of the main Type I tail are described in Part II. A classification of the tail forms according as the tail had the form of a fan of rays or a wedge, the orientation of the tail axis, and the angular width of the tail are discussed in Section IIA. In Section IIB the structure of the tail from 26 to 31 December, a period within which a disconnection of the tail took place, is viewed as the possible result of the passage across the comet of an interplanetary magnetic sector boundary, as proposed by Niedner and Brandt (1977, Bull. Amer. Astron. Soc. 4). In Sections IIC and IID waves in rays and the onset of turbulence are considered. Section IIIA is devoted to unusually long and bright off-axis rays; data on longitudinal forms, closing rate to the axis, and photometry of a ray are presented. In Section IIIB the structure of a "plume" of seven nearly parallel rays is related to the configuration of magnetic field lines predicted by Schmidt and Wegmann (1976, IPP Report 6/147). The orientations of the plume rays are consistent with those of the calculated field lines; the inferred rate of generation of the rays is about $1 / \mathrm{hr}$.
\end{abstract}

\section{INTRODUCTION}

Photographs of Comet Tago-SatoKosaka 1969 IX were made with the Michigan Curtis Schmidt $(0.6 \mathrm{~m}, \mathrm{f} / 3.5)$ on 12 nights in the period 25 December 1969 to 11 January 1970. In the course of the initial examination of these plates in 1970 , the writer was impressed by the comparatively simple patterns of the Type I tail. There was a notable absence of dramatic disruptions, such as were seen in the tail of Comet 1970 II several months later. No solar flares with Comprehensive Flare Indices equal to or greater than 8 occurred during the interval of our observations, whereas between 29 March and 6
April, when the tail of Comet 1970 II was disturbed, there were three such flares (Dodson and Hedeman, 1971, 1975). The purpose of this investigation is to examine the structure of a Type I tail during a period of seemingly orderly evolution, near maximum of solar cycle 20 .

The Michigan material includes twentyfour 2- to 5-min and one 1-min exposures on IIa-O emulsion. The comet was also observed concurrently with the Hamburg Schmidt at the Boyden Observatory $(0.36$ $\mathrm{m}, \mathrm{f1} / 1.7)$, using 103a-O emulsion. The two series of plates complement one another, and first positives on film have been exchanged. Particulars of the plates 
TABLE I

Plate Data, Tail Classes, and Tail Axis Orientations ${ }^{a}$

\begin{tabular}{|c|c|c|c|c|c|c|c|c|c|}
\hline Date $1969 / 70$ & Tel & $n$ & $\begin{array}{l}\text { Tail } \\
\text { class }\end{array}$ & $\theta$ & Date $1969 / 70$ & Tel & $n$ & $\begin{array}{l}\text { Tail } \\
\text { class }\end{array}$ & $\theta$ \\
\hline 25.04 Dec & M & 1 & $2 \mathrm{~A}$ & -3.0 & $4.81 \mathrm{Jan}$ & B & 1 & 2 & - \\
\hline 26.05 Dec & $\mathbf{M}$ & 3 & 1 & -1.0 & $5.79 \mathrm{Jan}$ & B & 1 & 2 & - \\
\hline 27.06 Dec & $\mathbf{M}$ & 2 & 1 & +1.0 & $7.80 \mathrm{Jan}$ & $\mathrm{B}$ & 1 & 2 & - \\
\hline 28.06 Dec & $\mathbf{M}$ & 3 & $2 \mathrm{~A}$ & -1.0 & 8.08 Jan & $\mathbf{M}$ & 2 & 2 & +3.0 \\
\hline $30.05 \mathrm{Dec}$ & $\mathbf{M}$ & 3 & 1 & -2.0 & 9.09 Jan & $\mathbf{M}$ & 1 & 2 & +2.0 \\
\hline $31.05 \mathrm{Dec}$ & $\mathbf{M}$ & 3 & 1 & -1.0 & 10.07 Jan & $\mathbf{M}$ & 3 & $2 \mathrm{~A}$ & +6.0 \\
\hline 1.54 Jan & $\mathrm{C}$ & 1 & 2 & 一 & $10.80 \mathrm{Jan}$ & $\mathrm{B}$ & 1 & 2 & - \\
\hline $2.06 \mathrm{Jan}$ & $\mathbf{M}$ & 3 & 2 & +3.5 & $11.10 \mathrm{Jan}$ & $\mathbf{M}$ & 1 & 2 & +3.0 \\
\hline 2.78 Jan & B & 1 & 2 & - & $12.78 \mathrm{Jan}$ & B & 1 & $2 \mathrm{~A}$ & - \\
\hline 3.79 Jan & B & 1 & 2 & - & & & & & \\
\hline
\end{tabular}

${ }^{a}$ Tel = telescope; $\mathbf{M}-$ Michigan ; B-Boyden; C-Candy (see text) $; n=$ number of plates or films; $\theta=$ position angle of tail axis relative to projection of Sun-comet radius vector.

and films will be found in Table I; several useful 5- and 20-sec Michigan IIa-O plates are not listed. It will be seen that from 25 December to 12 January there are three dates without a plate or film; a wellreproduced photograph by Candy (1970) on 1.54 January bridges one of these gaps.

Table II summarizes orbital data, and information on the aspect in which the comet was projected on the plates. The angle between the Sun-comet radius vector and the plane of the sky at the comet was not large enough to seriously foreshorten the image of the tail; in this work no corrections for foreshortening have been made. The cometocentric latitude of the Earth with reference to the orbit plane was also small (zero on 2 January) and we see the tail projected on a plane which was never far from perpendicular to the orbit.

It is well known that a Type I tail may undergo a complete alteration in appearance within $24 \mathrm{hr}$. Miller $(1969,1973)$ discussed a sequence of 18 Curtis Schmidt photographs of Comet 1957 III taken on the night of 5 May 1957. Marked changes in tail structure took place from plate to plate, that is, on a time scale of $20 \mathrm{~min}$, and the evolution of a new tail was followed for $6 \mathrm{hr}$. The present material does not provide comparable time resolution, and the combined set of Michigan and Boyden photographs is here regarded as providing

TABLE II

Geometrical Data

Perihelion passage ${ }^{a}$

Perihelion distance ${ }^{a}$

Inclination of orbit plane to solar equatorial plane

Range in geocentric distance of comet ${ }^{a}$

Range in heliocentric distance of comet ${ }^{a}$

Angle of Sun-comet radius vector to sky plane

Cometocentric latitude of Earth referred to orbit plane of comet

Earth in plane of comet orbit

Zenith distances at times of observations
21.27 December 1969

$0.473 \mathrm{AU}$

$69^{\circ}$

0.973 to $0.456 \mathrm{AU}$

0.482 to $0.720 \mathrm{AU}$

13 to 0 to $22^{\circ}$

9 to 0 to $24^{\circ}$

2.1 January 1970

69 to $84^{\circ}$

a Marsden, personal communication of daily ephemeris. 
instantaneous views of the comet at intervals of about 1 day.

The small scale of our plates, and competition by radiations from neutral molecules and a faint Type II tail do not permit resolution of plasma structures in the interesting region near the nucleus within which the components of the Type I tail are presumed to originate. The first of a series of Michigan photographs of the $\mathrm{C}_{2}$ coma made with an appropriate interference filter is a 5-min exposure taken on 11 January: The heavily exposed central region has a radius of $3.5 \times 10^{4} \mathrm{~km}$. On our Curtis Schmidt plates of 2- to 5 -min exposure, the Type I tail can be traced to within about $7 \times 10^{4} \mathrm{~km}$ of the photocenter; on the 5- and 20-sec exposures, this is reduced to 1.1 to $2.5 \times 10^{4} \mathrm{~km}$. We are therefore unable to offer any direct evidence bearing upon conflicting hypotheses concerning the place of origin of the tail plasma, and the manner in which it becomes segregated into rays.

\section{THE STRUCTURE OF THE MAIN TAIL}

A. Orientation, Classification, and Angular Width

The last column of Table I lists for the date of each Michigan plate, the angle $\theta$ between the projections on the sky of the tail axis and the Sun-comet radius vector. Uncertainty in the judgment of the position of the tail axis was of the order of $\pm 1^{\circ}$. Because of the low cometocentric latitude of the Earth (Table II), the usual characterization of the angle as preceding or lagging behind the orbital motion of the comet is meaningless, and it is signed positive or negative, as the position angle of the axis was greater or less than that of the projected radius vector. Prior to 2 January, $\theta$ was small and barely significant. On that date the Earth transited the orbit plane, and $\theta$ represents a deviation of the tail axis out of the orbit plane of about $3^{\circ}$ in the direction of solar rotation. This may be compared with $\pm 3^{\circ}$ found by Brandt and Hardorp (1970) for the dispersion in inclination of tail axes to orbital planes. Subsequent to 2 January, $\theta$ is positive and larger in magnitude; a comparable result is indicated by reproductions of Boyden plates of $5.79,7.80,10.80$, and 12.78 January (Jockers et al., 1972). Earlier than 2 January, if the tail axis lagged behind the radius vector in the plane of the orbit, the resulting projection on the sky would have made a negative contribution to $\theta$, opposite in sign to that of an inclination of the axis out of the plane in the direction of solar rotation. After 2 January both contributions would have been positive, which may account for the significantly positive observed values of $\theta$.

The preliminary examination of our plates in 1970 suggested a subdivision of the tails into two classes. On the later nights of the period of observation, the most common form consisted of a central tail having the shape described here as a "wedge," accompanied by short, faint off-axis rays. In the earlier part of the period, there are several nights when there was no primary wedge, and a fan of off-axis rays contributed as much to the visible tail as those on or near the axis. An elementary classification of the tail structure was thought to be useful for the present work, and the tails have been assigned to two classes, according as the primary wedge is absent (Class 1) or present (Class 2). A variant of Class 2 in which one or more bright, long rays lie outside of the wedge is designated Class 2A. The classes are listed in Table I and illustrated in Fig. 1.

A simple parameter of a tail is its angular width. Table III presents the data, defined as the angle subtended by the tail at the radius from the head given in the table. At such distances from the head, faint siderays are absent, and the borders of the tail, as determined by visual inspection, are unambiguous. Photometric cross sections of the kind described below 
FREEMAN D. MILLER

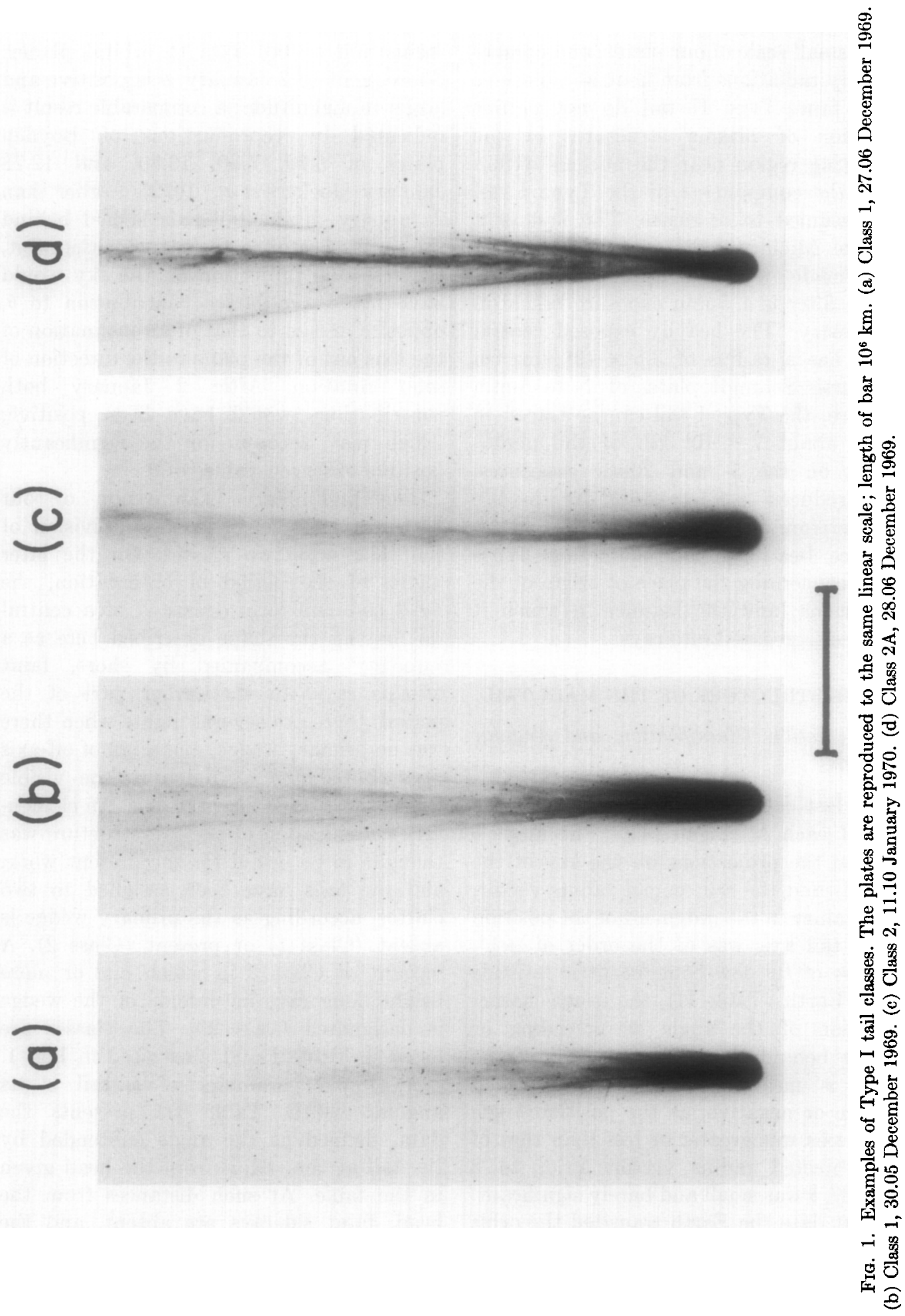


TABLE III

Angular Widths of Tail Fans and Wedges

\begin{tabular}{|c|c|c|c|c|c|}
\hline \multicolumn{3}{|c|}{ Class 1 fans } & \multicolumn{3}{|c|}{ Class 2 and $2 \mathrm{~A}$ wedges } \\
\hline Date & $\begin{array}{l}\text { Radius }^{a} \\
\left(10^{6} \mathrm{~km}\right)\end{array}$ & $\begin{array}{c}\text { Fan } \\
\text { Angle }^{b}\end{array}$ & Date & $\begin{array}{l}\text { Radius }^{a} \\
\left(10^{6} \mathrm{~km}\right)\end{array}$ & $\begin{array}{l}\text { Wedge } \\
\text { Angle }^{b}\end{array}$ \\
\hline $26.05 \mathrm{Dec}$ & 3.9 & 12.0 & 25.04 Dec & 3.6 & 10.5 \\
\hline 27.06 Dec & 4.9 & 6.5 & 28.06 Dec & 4.9 & 2.5 \\
\hline 30.05 Dec & 6.6 & 7.0 & $2.06 \mathrm{Jan}$ & 3.1 & 5.0 \\
\hline 31.05 Dec & 4.1 & 6.5 & $3.79 \mathrm{Jan}$ & 4.8 & 3.5 \\
\hline \multirow[t]{8}{*}{ Means } & 4.9 & 8.0 & 4.81 Jan & 7.1 & 5.0 \\
\hline & & & $5.79 \mathrm{Jan}$ & 7.5 & 3.0 \\
\hline & & & $7.80 \mathrm{Jan}$ & 10.5 & 4.0 \\
\hline & & & $8.08 \mathrm{Jan}$ & 2.5 & 3.0 \\
\hline & & & 10.07 Jan & 4.2 & 3.5 \\
\hline & & & $11.10 \mathrm{Jan}$ & 4.1 & 4.0 \\
\hline & & & 12.78 Jan & 6.7 & 2.0 \\
\hline & & & Means & 5.4 & 4.2 \\
\hline
\end{tabular}

${ }^{a}$ Distance from photocenter at which angle subtended by tail was measured.

${ }^{b}$ Measured to nearest half-degree.

for Comet 1957 III would doubtless lead to somewhat greater breadths. The widths are greater for Class 1 tails than for Class 2, as might be expected from the classification criteria.

Some years past, T. R. Dennis and the writer made photometric cross sections of the tail of Comet 1957 III from plates taken on 5 May 1957. The results were not published, but have been supplied to, and reproduced by Ershkovich (1976, Table I). From 0.8 to $3.4 \times 10^{6} \mathrm{~km}$, the linear width was essentially constant at $0.3 \times 10^{6} \mathrm{~km}$ (almost twice the breadth that would be estimated from visual inspection of the plates). Ershkovich (1976) concluded tentatively from this result that the magnetic field in this tail was primarily of external origin. On the other hand, the mean angular widths in Table III (8 and $4^{\circ}$ for Classes 1 and 2, respectively) are in good agreement with Ershkovich's prediction of 5 to $7^{\circ}$ calculated for typical plasma parameters. In this case, an intrinsic component of the magnetic field in the tail could be comparable to that captured from the solar wind (Ershkovich, private communication, 1977).
An alternative and nonmagnetic interpretation of our tail angle data is provided by the theory of Wallis (1968). The tail of Comet 1969 IX would correspond to the case in which envelopes of hot plasma generate ray systems as they sink toward the nucleus from its sunward side. Comet 1957 III is an example of a single axial tail fed by cold plasma in which ionization had ceased, and which flowed straight through the head into the tail.

\section{B. Class 1 Tails and the Period 26 to 31 December}

Figures $1 \mathrm{a}$ and $1 \mathrm{~b}$ illustrate types of ray patterns that we have grouped in Class 1 . Figure 2 is a reproduction of a 20-sec exposure taken half an hour before the plate of Fig. 1a, depicting details of the tail closer to the nucleus than does the longer exposure. On the original plate, the structure typical of Class 1 can be traced to within $19000 \mathrm{~km}$ of the nucleus. On a 5-sec exposure of 30 December, the three brightest rays in Fig. $1 \mathrm{~b}$ are resolved to $57000 \mathrm{~km}$ from the photocenter.

The observed configurations are the two- 


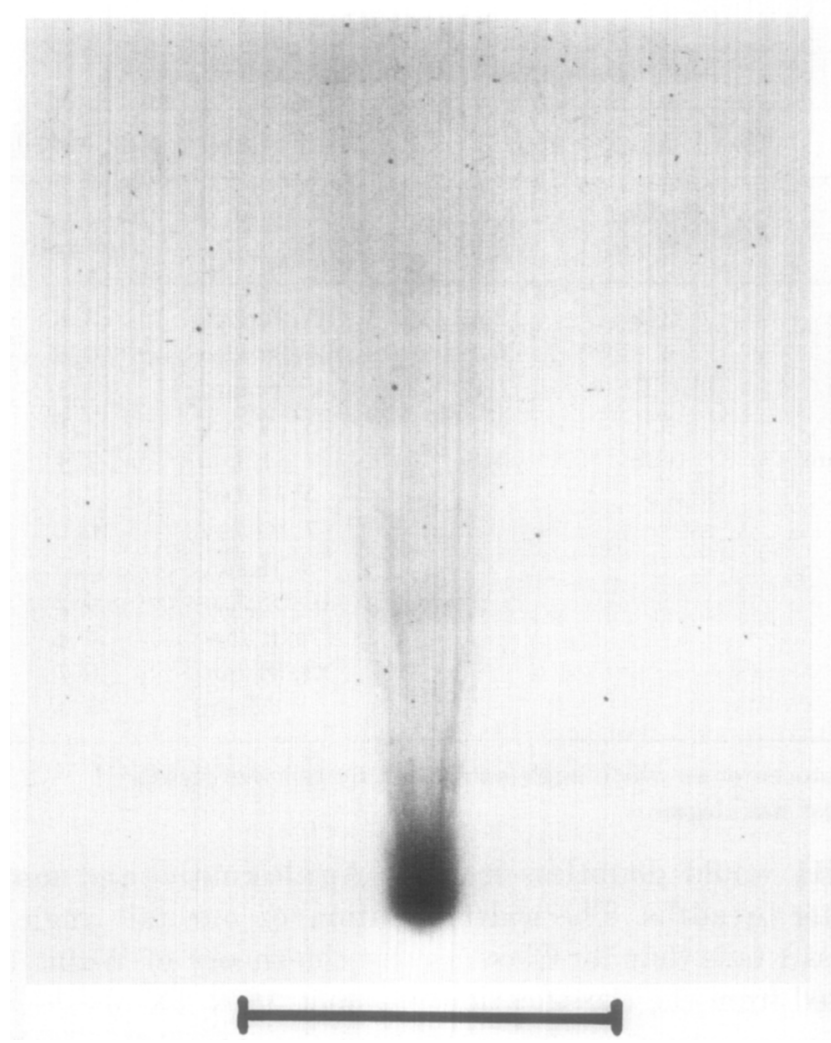

Fia. 2. Class 1, 27.04 December 1969, 20-sec exposure, length of bar $5 \times 10^{5} \mathrm{~km}$.

dimensional projections of rays distributed in azimuth about the axis, and off-axis rays near the plane containing the Earth and axis would project close to the latter. The apparent dearth of axial rays must therefore reflect a real deficiency in three dimensions, corresponding to the canonical description of Wurm (1963), in which rays fade out as they fold toward the axis.

Lacking an extended sequence of plates on each night, it is still possible to draw inferences concerning the duration of the pattern of plasma flow that gives rise to Class I tails. An elementary calculation based on the flow diagrams derived by Jockers et al. (1972) suggests that the development of the visible length of the Class 1 tails would require 18 to $24 \mathrm{hr}$, and it is also relevant that the Class 1 pattern is seen on two pairs of consecutive nights, 26, 27 and 30,31 December.
Current speculation about the role of magnetic fields in tail development has led us to search for any magnetic phenomenon of the solar wind that might be associated with the appearance and subsequent disappearance of the Class 1 characteristic on four of the five nights from 26 to 31 December for which we have plates. On 28 December the tail was classed as $2 \mathrm{~A}$ (Fig. 1d) but on that night we see an example, unique in our set of plates, of a wedge to which the supply of plasma has been cut off. Starting at the photocenter, there is no visible trace of the wedge to a distance of $0.9 \times 10^{6} \mathrm{~km}$, at which point two thread-like filaments appear. At 1.3 $\times 10^{6} \mathrm{~km}$ these blend into an increasingly bright wedge, which is well developed from $1.6 \times 10^{6} \mathrm{~km}$ to the edge of the plate at $6.9 \times 10^{6} \mathrm{~km}$. Further evidence for intermittent plasma supply is provided by the 
discontinuous structure of the western Class $2 \mathrm{~A}$ ray on this date. It is bright to a distance of $1.8 \times 10^{6} \mathrm{~km}$ from the photocenter, fainter to $2.6 \times 10^{6} \mathrm{~km}$, brighter to $3.1 \times 10^{6} \mathrm{~km}$, and then becomes fainter and irregular.

Niedner and Brandt (1977) suggest that disconnection of a tail may be caused by passage of a magnetic sector boundary. The latitude of the comet decreased from $-65^{\circ}$ on 26 December to $-52^{\circ}$ on 31 December, and the high latitude structure of sector boundaries is uncertain. Smith and Tsurutani (1978) analyzed Pioneer 11 data in terms of a model in which the sector boundary corresponds to a current sheet surrounding the Sun, and inclined less than $90^{\circ}$ to the plane of the solar equator, finding the inclination to be only about $15^{\circ}$. However, they point out that their data refer to sunspot minimum, and Svalgaard and Wilcox (1976) deduced from cosmic ray observations near maximum that magnetic field structure may extend to at least latitude $45^{\circ}$.

Hedgecock (1975) concluded from Heos satellite data that a sector boundary crossed the Earth on 5.3 January 1970. Extrapolation by one solar rotation of a sector boundary that Wilcox and Colburn (1972) found from Explorers 33 and 35 records to persist throughout 1969 also yields Earth transit on that date. Assuming that the boundary corresponds to a current sheet, it must have been inclined $61^{\circ}$ to the plane of the solar equator if it crossed the comet on the date of tail disconnection on 28 December. If it encountered the comet about 26 December, at the onset of Class 1 tail structure, the inclination would have been $65^{\circ}$. As a result of the comet's motion in heliographic latitude and longitude, a sector boundary inclined $65^{\circ}$ to the solar equator would have advanced with respect to the comet at a mean rate of $1.7 /$ day between 26 and 31 December. It should be interesting to examine other instances in which a sector boundary may have travelled across a comet at low relative angular velocity.

\section{Waves}

Ershkovich and Chernikov (1973) have derived wave parameters of sinuous rays of this comet from illustrations of Boyden plates (Jockers et al., 1972), so we confine ourselves to the Michigan photographs.

It is not difficult to identify rays with wavelike characteristics, provided that one is content with not more than two complete cycles (e.g., Miller, 1969; Brandt, 1976). Of the seven nights when rays of suitable length were well defined, one or two undulatory forms were present on four, but no instances of more than two cycles were found. In the absence of plates separated by a time interval adequate for meaningful measurements of the velocities of these features, further discussion would be unprofitable.

The presence or absence of parallel undulations in adjacent rays may be a clue to the mechanism of ray generation. Ershkovich (1977) discussed the instabilities of a cylindrical plasma tail with a diameter of several times $10^{5} \mathrm{~km}$. The boundary of the cylinder may undergo Kelvin-Helmholtz instability, the amplitude of the resulting waves decreasing only slowly toward the axis. Waves well inside the cylinder should be indicative of conditions at the boundary. The cylinder may, however, break up into independent tails, of which the long bright off-axis rays of our Class 2A may be examples (Ershkovich, private communication 1977), and coordinated waves are not then anticipated. In the theory of Wallis (1968) each ray is stabilized independently by the supersonic flow of the solar wind, and parallel undulations would not be expected.

Parallel waves are indeed observed. Wolf (1909) stated that the tail of Comet 1908 III often exhibited four or more rays oscillating in parallel. A more recent example was provided by Comet $1957 \mathrm{~V}$; 
on 18 August 1957, the rays forming the main tail undulated together (Miller, 1958, Plate Ia).

We have searched the Michigan plates of Comet 1969 IX for such coordinated waves and find no convincing examples. The amplitudes of the undulations are small, and we do not consider that we can discriminate on these grounds between a single "monolithic" tail and one composed of independent rays.

\section{Turbulence}

The possibility that Kelvin-Helmholtz instability may lead to turbulence in the far tail is a matter of debate. Ershkovich (1976) expects the tail to become a turbulent wake, whereas Mendis (1977) considers that turbulence is seldom observed, and reserves judgment on the theoretical aspect.

Observationally, turbulence is difficult to define and measure. On plates of five dates, Jockers et al. (1972) identified numerous small features in the tail of Comet 1969 IX that were sufficiently well defined to permit measurement for velocity displacements - evidence of structural irregularities. We do not, however, class these small-scale inhomogeneities as turbulence. For example, on 10.07 January, velocities were derived for 44 features on the Michigan plates, lying between 0.5 and $4.0 \times 10^{6} \mathrm{~km}$ from the head (Jockers et al., 1972, Figs. 1c and 2c). But the main tail is reasonably orderly to its visible limit of $4.5 \times 10^{6} \mathrm{~km}$, and the two bright off-axis rays characteristic of our Class $2 \mathrm{~A}$ are straight and unbroken to $2.9 \times 10^{6} \mathrm{~km}$. We do not call this a turbulent tail. The tail on a Boyden plate of 10.80 January (Jockers et al., 1972, Fig. 1d) is described by the authors as having "an especially richly structured, almost turbulent aspect," and we classify it as turbulent.

It should be kept in mind that tail disturbances may be due to external causes such as interplanetary shocks (e.g., Jockers
TABLE IV

Onset of Turbulence

\begin{tabular}{cc}
$\begin{array}{c}\text { Date } \\
(1970)\end{array}$ & $\begin{array}{c}\text { Distance from } \\
\text { photocenter } \\
\left(10^{6} \mathrm{~km}\right)\end{array}$ \\
\hline 2.06 Jan & 3.7 \\
2.78 Jan & 2.5 \\
3.79 Jan & 4.4 \\
10.80 Jan & 2.6 \\
11.10 Jan & 3.1 \\
12.78 Jan & 5.4 \\
Mean & 3.6 \\
\hline
\end{tabular}

and Lust, 1973), and that if, as discussed in the preceding section, some rays can be treated as independent tails, turbulence may appear in one ray and not in another.

Recognizing that the identification of turbulence in the tails lacks rigor, we list in Table IV the dates when the tail was deemed sufficiently chaotic for the term "turbulence" to be merited, and, in the second column, the distance from the photocenter at which the onset of turbulence becomes obvious.

\section{OFF-AXIS RAYS}

\section{A. Off-Axis Rays of Class 2.A}

The term "off-axis" is used here to designate rays that lie distinctly outside the main fan or wedge of the tail. In this section we discuss the especially long and bright off-axis rays which characterize Class $2 \mathrm{~A}$ tails. Table $\mathrm{V}$ presents a variety of data on these rays. The tabulated lengths are of course at the mercy of the characteristics of the telescopes and the exposure times. The plate of 25 December was only exposed for $1 \mathrm{~min}$, but it may be compared with a 20-sec exposure on 28 December. The main tail is about the same length on both plates, but the $2 \mathrm{~A}$ rays on the 25 December exposure are only half the length of those of 28 December.

The western ray of 28 December is discontinuous, as though the plasma supply 
TABLE V

Class 2A Off-Axis Rays

\begin{tabular}{cccccccc}
\hline Date & \multicolumn{4}{c}{ Observed $^{a}$} & & \multicolumn{2}{c}{ Rectified $^{b}$} \\
\cline { 2 - 5 } & $(1)$ & $(2)$ & $(3)$ & $(4)$ & & $(5)$ & $(6)$ \\
\hline 25.04 Dec 1969 & 1.6 & 8,8 & - & - & & - \\
28.06 Dec 1969 & 6.5 & 3,7 & +0.2 & -1.3 & & +0.3 & -1.4 \\
10.07 Jan 1970 & 3.2 & 4,5 & -1.4 & -1.2 & & -2.0 & -1.3 \\
12.78 Jan 1970 & 9.0 & 4 & - & - & & - \\
\hline
\end{tabular}

a (1) Mean length $\left(10^{6} \mathrm{~km}\right)$; (2) angles of rays to tail axis $\left({ }^{\circ}\right)$; (3) mean angular rate of rays $\left({ }^{\circ} / \mathrm{hr}\right)$; and (4) predicted angular rate $(\% / \mathrm{hr})$ (Wurm, 1972).

${ }^{b}(5),(6)$ same as (3), (4), respectively, but rectified to assumed angle of $45^{\circ}$ between plane of ray and plane of sky.

- One ray.

had been turned on and off (Section IIB). We have searched for remnants of such rays distant from the head, which might be instances of earlier cutoff of plasma supply, but find none.

Wurm (1963) has remarked on the tendency for rays to occur in pairs symmetrical with respect to the axis. This is the case on three of the four nights when a $2 \mathrm{~A}$ tail was present. The phenomenon is especially noteworthy here because of the length and brightness of the rays, and the roughly equal angles made with the tail axis (Table V, col. 2).

The longitudinal forms of the four rays of 28 December and 10 January have been examined, using contact prints. Seven to 12 points were chosen on each ray, at distances from the photocenter ranging in the mean from 0.8 to $4.3 \times 10^{6} \mathrm{~km}$. After unprofitable trials of parabolae and hyperbolae, straight lines were fitted by least squares; departures of the measured points from the lines are unsystematic and small, averaging $\pm 1.5 \times 10^{4} \mathrm{~km}( \pm 0.3 \mathrm{~mm}$ on the plates). The lines intersect the tail axis from $3.2 \times 10^{6} \mathrm{~km}$ sunward to 0.5 $\times 10^{6} \mathrm{~km}$ antisunward of the nucleus.

Turning of rays toward the axis is a familiar phenomenon (Wurm 1963). The Michigan plates are not well suited for study of the turning rate (plate pairs have time intervals of 0.28 and $0.53 \mathrm{hr}$ on 28 December and $0.53 \mathrm{hr}$ on 10 January) but because of the length and straightness of the rays, it was thought worth a trial. The results are given in Table $\mathrm{V}$, col. 3 ; the estimated uncertainty in the rates is $\pm 0: 3 / \mathrm{hr}$. Observed rates are physically meaningful only if the plane containing ray and nucleus lies in the plane of the sky. To illustrate the sensitivity to this assumption, the rates have been rectified for an assumed angle of the ray plane to the sky of $45^{\circ}$ (col. 5). The expected rates are small for rays lying so close to the axis; cols. (4) and (6) are the rates predicted by the interpolation formula of Wurm and Mammano (1972, Fig. 3).

In their study of motions in the tail of this comet, Jockers et al. (1972) specifically refer to velocities in the $2 \mathrm{~A}$ rays of 10.07 and 12.78 January. In these three rays the velocities are described as increasing with distance from the head $(160 \mathrm{~km} / \mathrm{sec}$ at $5 \times 10^{6} \mathrm{~km}$ on 12.78 January), whereas near the axis the velocities were lower, and did not increase with distance as systematically as in the rays. The authors state that the motions were in all cases directed parallel, or nearly parallel to the tail axis, but the orientations of the longer plotted velocity vectors are consistent with motion parallel to the $2 \mathrm{~A}$ rays. 


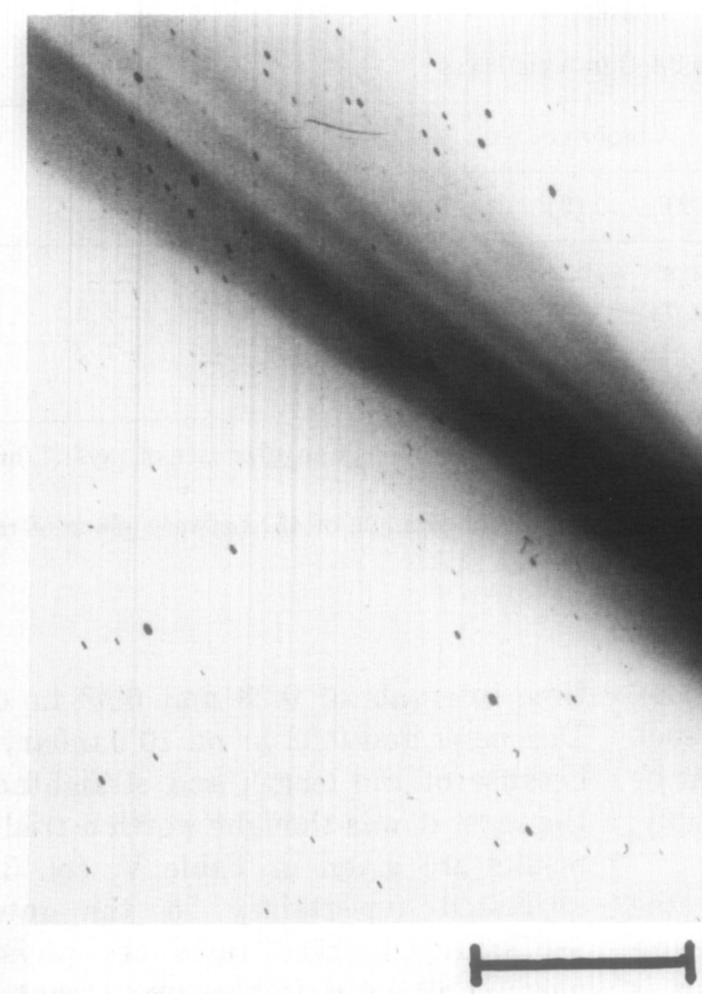

Fig. 3. 2.06 January 1970. Length of bar $2 \times 10^{5} \mathrm{~km}$.

The appearance of Class $2 \mathrm{~A}$ rays demonstrates that they are not elementary structures. They may widen, and at distance from the head, resolve into two or more components. To investigate this matter in detail, the northeasterly ray of 10 January has been studied by Clifford Arnold. Tracings perpendicular to the ray were made with the microdensitometer of the McMath-Hulbert Observatory at 20 points between 0.46 and $2.74 \times 10^{6} \mathrm{~km}$ from the photocenter. At 17 positions three tracings, and at the remaining three points two tracings were made. At each position the tracings are, of course, almost identical, but provide material for drawing independently smoothed ray profiles, which were averaged to obtain the information desired.

At $10^{6} \mathrm{~km}$ from the photocenter the profiles begin to show symptoms of multiple structure, which at successively greater distances resolves into two, and then into three partially overlapping rays of different surface brightness. We have decomposed these profiles into two or three components by assuming that each ray was symmetrical about its own axis, and that on the side opposite the two fainter rays, the brightest ray was unblended.

The brightest component, freed from the influence of its two companions, widens with increasing distance from the head, subtending an angle of $1^{\circ} .2 \pm 0^{\circ} .2$ (m.e.), and is slightly more than twice as wide as would be estimated from visual inspection of the plate. The fainter rays make angles of 1 and $2^{\circ}$, respectively, with the brightest component.

These observations signal caution, if space densities of tail ions are to be derived from measured surface brightnesses of rays. Inspected visually, the ray described above appears single to $1.3 \times 10^{6} \mathrm{~km}$ 


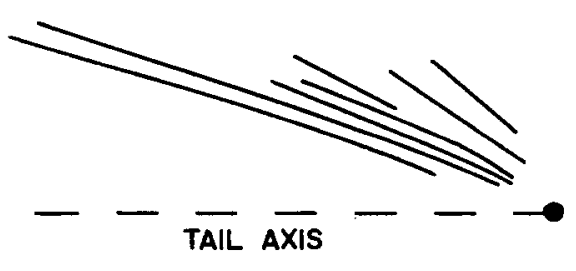

$200,000 \mathrm{KM}$

Fig. 4. The "plume," 2.06 January. Compare with Fig. 3.

from the photocenter, but, on the evidence presented above, it is composed of at least three rays. If the ions contributing to the photographic image are concentrated in several filaments, the densities will be greater than would be the case if they were dispersed throughout the apparent cross section of the unresolved structure.

\section{B. Shorter Off-Axis Rays}

Off-axis rays shorter and fainter than those of Class 2A, do not exhibit a significant degree of symmetry about the axis, lack of which is prominent on 2 January, when the comet had developed to the east of the main tail a rich system of rays. This array is illustrated (insofar as the steps in reproduction permit) in Fig. 3. Seven rays (referred to henceforward as the "plume") lie furthest from the axis. Closer to the axis is a compact group of four or five poorly resolved rays, and finally, two bright diffuse rays can be traced to $1.5 \times 10^{6} \mathrm{~km}$ from the head. The structure is asymmetrical, no rays are visible to the west of the main tail. A configuration in the tail of Comet $1957 \mathrm{~V}$ similar to the plume is described by Lüst (1962) as a "bundle" of numerous parallel rays. It was seen on 22 August (Lüst, Fig. 1) and had disappeared by the following night. No plume appears on the opposite side of the axis.

The rays of 2 January were traced on prints of different exposures at 10-fold enlargement, and a composite produced (Fig. 4). The orderly arrangement suggests that the rays were not generated at random in time and space-limitation of the rays on this date to one side of the axis required that development be confined to at most a sector of $180^{\circ}$ in azimuth about the axis. The plume is therefore especially suitable for comparison of the observed rays with the predictions of a model of plasma flow in cometary tails. We shall suppose that the rays of the plume lie very nearly in the same plane. There is no compelling evidence for this assumption, but Fig. 4 shows that the lengths of the rays increase with decreasing angle to the axis as described by Wurm (1963, Fig. 1). If the plume rays were distributed at random about the axis, one might expect to find short "young" rays projected closer to the axis than "older" more evolved rays, which is not the case.

A plasma tail model that predicts explicitly the forms of rays as a function of time is that of Schmidt and Wegmann (1976). Dr. Schmidt has provided an enlargement of Fig. 1a of the paper cited that is more suited to our needs, and on which magnetic field lines (along which plasma rays might form) are identified with the elapsed times ("ages") since they encountered the bowshock. The exact shape of the field lines is a function of the assumed interplanetary strength and the cometary gas production rate, taken by the authors for the purpose of the calculations as $5 \gamma$ and $2 \times 10^{30}$ molecules/sec, respectively.

Since the rays in our Fig. 4 are so nearly rectilinear, we have not attempted to compare details of their curvature with the model. Instead, the plume rays were superposed on the model diagram, and each observed ray was associated with the model field line which crossed the midpoint of the former. The parameters used to compare observation and model are the slopes $\eta_{0}$ and $\eta_{\mathrm{m}}$ of the observed ray and its associated model field line with respect to the axis, both averaged over the observed ray segment.

The model diagram represents the plane 
TABLE VI

Comparison of Off-AXis Rays with the Model of Schmidt and Wegmann

\begin{tabular}{|c|c|c|c|c|c|c|c|}
\hline \multicolumn{4}{|c|}{ A. 2 January } & \multicolumn{4}{|c|}{ B. 28 December } \\
\hline$\phi$ & $\left\langle\eta_{0}-\eta_{m}\right\rangle^{a}$ & $\langle\Delta t\rangle$ & $\iota_{\mathbf{d}}$ & $\phi$ & $\left\langle\eta_{0}-\eta_{m}\right\rangle^{a}$ & $\Delta t$ & $t_{\mathrm{d}}$ \\
\hline $0^{\circ}$ & $+1^{\circ}\left( \pm 1^{\circ}\right)$ & $1^{\mathrm{b} 3}$ & 8.0 & $0^{\circ}$ & $0^{\circ}\left( \pm 4^{\circ}\right)$ & $11^{\mathrm{h}} 4$ & $7^{\mathrm{h}} \cdot 4$ \\
\hline 30 & $+1( \pm 1)$ & 1.2 & 7.4 & 30 & $+3( \pm 3)$ & 1.0 & 6.9 \\
\hline 45 & $+4 \quad( \pm 2)$ & 1.0 & 6.6 & 45 & $+7( \pm 2)$ & 0.6 & 6.3 \\
\hline 60 & $+9( \pm 4)$ & 0.8 & 5.3 & 60 & $+13 \quad( \pm 3)$ & 0.4 & 5.1 \\
\hline
\end{tabular}

a Figures in parentheses are averages of the differences between individual values for the rays and the preceding mean.

containing field line and tail axis whereas the plane of an observed ray is inclined at an unknown angle $\phi$ to that of the sky; we have rectified the observed rays for assumed values of $\phi$ of 30,45 , and $60^{\circ}$. The inclination of the tail axis to the sky plane is also unknown, but since the inclination of the Sun-comet radius vector to the sky was only $9^{\circ}$, we have assumed the tail axis inclination to be zero.

The tail axis drawn in Fig. 4 is that appropriate to the full length of the observed tail; it represents an average axis evolved over a greater period of time than that represented by the plume rays. We have therefore made use of a 5-sec exposure to define the axis between 0.5 and $3 \times 10^{5}$ $\mathrm{km}$ from the photocenter, and find it to lie $3^{\circ}$ nearer the plume than the axis of Fig. 4.

Comparison of the observed and associated model rays was carried through for the two choices of the tail axis described above. The difference in $3^{\circ}$ of the axis is largely compensated by a change in the field line with which each plume ray is associated, and in Table VIA we present only the results that depend on the axis of the inner $300000 \mathrm{~km}$ of the tail. The second column gives the mean for the seven rays of the differences in slope of the observed and associated model rays. Each figure in parentheses is the average deviation of the seven rays from the preceding mean, and is not an error dispersion.
The rate at which rays are generated is linked by Schmidt and Wegmann (1976) to the frequency with which tangential discontinuities in the solar wind encounter the comet, that is, about $1 / \mathrm{hr}$ (Burlaga, 1971). For comparison with this datum we have differenced the "ages" of the model field lines associated with the plume rays. The mean intervals $\langle\Delta t\rangle$ are entered in the third column of Table VIA; the following column lists the "age" $t_{\mathrm{d}}$ of the plume ray most distant from the axis.

On the plates of 28 December, two short rays lie to the east of the axis. The outer ray is the only example on the Michigan plates of an instance in which the inner visible tip lies as far sunward as the perpendicular to the axis through the photocenter. The rays were fitted to the model of Schmidt and Wegmann, following the same procedure that was employed for the plume of 2 January, with the results shown in Table VIb, which are consistent with those for the plume.

The entries in Table VI confirm the compatability of the observed rays with the model of Schmidt and Wegmann, provided that the inclinations of the ray planes to the sky did not significantly exceed $45^{\circ}$, for which the a priori probability is 0.5 . It is inferred that on $1 / 2$ January, for a period of about $6 \mathrm{hr}$, rays were generated at approximately $1 / \mathrm{hr}$, in agreement with the rate explained by Schmidt and Wegmann as the result of 
encounters by the comet with tangential discontinuities in the solar wind.

The preceding discussion suggests that the rays of the plume can be viewed as depicting seven stages in the evolution of a single hypothetical ray. If this is correct, the inclinations $(\eta)$ of the rays to the tail axis, and their "ages" (as derived above) should combine to give rates of change of inclination consistent with observed closing rates of rays. For the latter we depend upon Fig. 3 of Wurm and Mammano (1972) which incorporates 19 rates derived from observations of eight comets, represented by the interpolation formula $d \eta / d t$ $=\exp (\eta / 20)$. Since the measurements of Wurm and Mammano could not be rectified for inclination of the ray planes to the sky, we use our unrectified data. By differencing the inclinations and "ages" of successive pairs of ray plumes, we obtained six rates as a function of inclination. Because the interpolation formula of Wurm and Mammano predicts a rate of $1 \% / \mathrm{hr}$ on the axis, we have preferred the relation $d \eta / d t=\exp (\eta / C)-1$ and find the best fit for $C=18$. This predicts rates varying from $6.1 / \mathrm{hr}$ for the outer pair of rays at mean inclination $35^{\circ}$, to $1: 5 / \mathrm{hr}$ for the inner pair at inclination $16^{\circ}$; the six points scatter about the predicted curve with an average deviation of $\pm 1.2 / \mathrm{hr}$. Our relation lies within the range bars which enclose the scatter of the individual points that contributed to the figure of Wurm and Mammano. This result supports our interpretation of the plume as the result of the orderly development of a system of nearly coplanar rays.

\section{ACKNOWLEDGMENTS}

The author is grateful to Rh. Lüst and K. Jockers for providing film copies of the Boyden plates, to H. W. Dodson for making available the microdensitometer of the McMath-Hulbert Observatory, to H. U. Schmidt for his diagram of theoretical magnetic field lines, and to A. I. Ershkovich for a lively correspondence on some aspects of models of plasma tails. This work was supported in part by the National Science Foundation.

\section{REFERENCES}

Brandt, J. C., ANd Hardorp, J. (1970). Ionic comet tails and the direction of the solar wind. Astron. Astrophys. 5, 322-324.

BrandT, J. C. (1976). Review-observations of recent comets-ion tails. In The Study of Comets,. Proc. I.A.U. Colloq. No. 25 (B. Donn, M. Mumma, W. Jackson, M. A'Hearn, and R. Harrington Eds.), pp. 361-366. NASA SP-393.

BurlagA, L. F. (1971). Nature and origin of directional discontinuities in the solar wind. $J$. Geophys. Res. 76, 4360-4365.

Candy, M. P. (1970). Observer's page. Sky \& Tel. 39, 263.

Dodson, H. W., and Hedeman, E. R. (1971). An experimental, comprehensive flare index and its derivation for "major" flares, 1955-1969. World Data Center A, Report UAG-14.

Dodson, H. W., and Hedeman, E. R. (1975). Experimental comprehensive solar flare indices for certain flares, 1970-1974. World Data Center A, Report UAG-52.

Ershrovich, A. I. (1976). On the origin of the magnetic field in Type-I comet tails. Mon. Not. Roy. Astron. Soc. 177, 649-653.

Ershrovich, A. I. (1977). Solar Wind Interaction with Type-I Comet Tails. In Study of Travelling Interplanetary Phenomena/1977 (M. A. Shea, D. F. Smart, and S. T. Wu, Eds.), pp. 305-322, D. Reidel, Dordreeht.

Ershkovich, A. I., AND Chernikov, A. A. (1973). Non-linear waves in Type-1 comet tails. Planet. Space Sci. 21, 663-670.

HedGeCock, P. S. (1975). The heliographic latitude dependence and sector structure of the interplanetary magnetic field 1969-1974: Results from the Heos satellites. Solar Phys. 44, 205-224.

Jockers, K., AND Lüst, RH. (1973). Tail peculiarities in Comet Bennett caused by solar wind disturbances. Astron. Astrophys. 26, 113-121.

JockmRs, K., Lüst, RH., and NowaK, Th. (1972). The kinematical behavior of the plasma tail of Comet Tago-Sato-Kosaka 1969 IX. Astron. Astrophys. 21, 199-207.

Lüst, RH. (1962). Die Bewegung und Form von Strukturen im Schweif des Kometen Mrkos 1957d. Z. Astrophys. 54, 67-97.

Mendis, D. A. (1977). On the origin of the magnetic field in Type-1 comet tails: A reply to Ershkovich. Mon. Not. Roy. Astron. Soc. 179, 5P-8P.

Miller, F. D. (1958). Filters for comet photography-Comet Mrkos, 1957d. Publ. Astron. Soc. Pac. 70, 279-284.

Miller, F. D. (1969). Comet Arend-Roland (1957 III) on May 5, 1957. I. Development and kinematics of the Type-I tail. Astron. J. 74, 248-255. MILLER, F. D. (1973). Comet Arend-Roland (1957 
III) on May 5, 1957. II. Transient structures in the Type-I tail. Astron. $J .78,1125-1130$.

Niedner, M. B., and Brandt, J. C. (1977). Plasma disconnection events in comets and magnetic reconnection at sector boundaries. Bull. Amer. Astron. Soc. 4, 618.

Schmidt, H. U., and Wegmand, R. (1976). Interaction of interplanetary magnetic fields with cometary plasma. In 2nd European Conf. on Computational Physics (D. Biskamp, Ed.), IPP Report 6/147.

Smith, E. J., and Tsurutani, B. T. (1978) . Observations of the interplanetary sector structure up to heliographic latitudes of $16^{\circ}$ : Pioneer 11. $J$. Geophys. Res. 83, 717-724.

SvalgaARd, L., and Wilcox, J. M. (1976). Structure of the extended solar magnetic field and the sunspot cycle variation in cosmic ray intensity. Nature 262, 766-768.

Wallis, M. K. (1968). The fountain model for plasma envelopes of comets. Planet. Space Sci. 16, 1221-1248.

Wilcox, J. M., and Colburn, D. S. (1972). Interplanetary sector structure at solar maximum. $J$. Geophys. Res. 77, 751-756.

Wolf, M. (1909). Utber den Schweif des Kometen 1908c (Morehouse). Astron. Nachr. 180, 1-12.

WurM, K. (1963). The physics of comets. In The Moon, Meteorites and Comets (B. M. Middlehurst and G. P. Kuiper, Eds.), pp. 573-617. Univ. of Chicago Press.

Wurm, K., and Mammano, A. (1972). Contributions to the kinematics of Type-I tails of comets. Astrophys. Space Sci. 18, 273-286. 